\title{
Scaling of conductivity and Young's modulus in replicated microcellular materials
}

\author{
Andreas Mortensen - Yves Conde • Andreas Rossoll • \\ Christopher San Marchi
}

Received: 12 April 2013/Accepted: 24 July 2013/Published online: 14 August 2013

(C) Springer Science+Business Media New York 2013

\begin{abstract}
Scaling exponents for the conductivity and stiffness of replicated microcellular materials exceed commonly predicted values of 1 and 2 . We show here that this is caused by the fact that, in replicated microcellular materials, the solid architecture varies with the relative density: a simple derivation based on the physics of powder consolidation returns and explains the observed scaling behaviour. The same derivation also gives an explanation for Archie's law, known to describe the conductivity of wet soils.
\end{abstract}

\section{Introduction}

The thermal or electrical conductivity $C$ and the Young's modulus $E$ of microcellular materials vary strongly with their relative density $V_{\mathrm{m}}$. When solid-phase contributions are dominant, this dependence is generally described using a power-law scaling relation [1]:

$\frac{C}{C_{\mathrm{m}}}=k\left(V_{\mathrm{m}}\right)^{n}$ and $\frac{E}{E_{\mathrm{m}}}=K\left(V_{\mathrm{m}}\right)^{N}$

where $C_{\mathrm{m}}$ is the conductivity and $E_{\mathrm{m}}$ is the Young's modulus of the constitutive solid material making the

A. Mortensen $(\bowtie) \cdot$ Y. Conde · A. Rossoll

Laboratory of Mechanical Metallurgy, Ecole Polytechnique

Fédérale de Lausanne (EPFL), 1015 Lausanne, Switzerland

e-mail: andreas.mortensen@epfl.ch

Present Address:

Y. Conde

Constellium, EPFL Quartier de l'Innovation, Bât. E,

1015 Lausanne, Switzerland

C. San Marchi

Sandia National Laboratories, Livermore, CA 94550, USA porous material, while $k, n, K$ and $N$ depend on the porous material's mesostructure or architecture (i.e. the geometry of solid around the pores). In microcellular materials made of identical interconnected straight struts of uniform cross section, analysis predicts $n=1$ and $N=2$ for highly porous structures, in which $V_{\underline{m}}$ is well below unity [1-3].

There are many ways of making microcellular materials; among them replication processing is one of the most convenient if an open-pore material is desired [4-9]. Replication processing begins with the production of a porous 'preform' made of packed and densified particles of a soluble refractory space-holder material. A melt or slurry is then infiltrated into open pores of the preform, and solidified. Finally, the refractory space-holder material is removed by leaching. The resulting 'replicated' microcellular solid contains a network of interconnected, open, pores; as the name of the process indicates, its structure is a replication of the open pore space between densified spaceholder particles that made the soluble preform. Advantages of the process include its simplicity, as well as its ability to produce relatively regular microcellular structures of metal, polymer or ceramic, with independent control of the pore volume fraction, size and shape.

For both replicated and other microcellular materials, the scaling exponent $n$ in the relationship for the conductivity (Eq. 1) is seldom 1: observed $n$ values generally range between 1.5 and 1.8 [1, 2, 10]. Equation (1) with $n \approx 1.5-1.8$ is also found in experimental data from similar structures, namely (i) metal matrix composites containing a high volume fraction of electrically insulating particles [1113] and (ii) fluid-saturated rock and soil, where electricity is carried by ionic conduction through liquid filling the space between consolidated insulating particles; in soil science, this is known as 'Archie's law' [14-17]. Various interpretations have been offered for observed $n$ values; these 
include continuum mean-field approximations (among which the differential effective medium model indeed returns $n \geq 1.5$ and accounts for the influence of particle shape, but does not explain the law physically), as well as the view that this exponent is a manifestation of percolationbased scaling around a percolation threshold situated at or near $V_{\mathrm{m}}=0$ [3, 10, 14-22].

Mechanical testing of most open or closed cell microcellular materials gives values of the exponent $N$ in the scaling law for Young's modulus (Eq. 1) situated between 1 and 2. This is in keeping with models, both mean-field and numerical, of the elastic stiffness of microcellular materials, essentially all of which predict $N \leq 2[1-3$, 23-34]. With replicated microcellular materials, on the other hand, one finds $N \approx 2.6-3$, and the value of $N$ tends to increase as $V_{\mathrm{m}}$ decreases $[23,35]$. To our knowledge, Roberts and Garboczi [34] have proposed the only model in the literature that produces $N$ so clearly in excess of two; however, the corresponding ('Gaussian Random Field') structure differs fundamentally from that of replicated microcellular materials. So this model does not offer a physical explanation.

We propose here that the physical origin of the experimentally observed scaling exponents of replicated microcellular materials for both conductivity $(n \approx 1.5-1.8)$ and for Young's modulus $(N \approx 2.6-3.0)$ lies in the fact that their mesostructure does not remain geometrically selfsimilar as $V_{\mathrm{m}}$ varies. To show this, we give in what follows a simple derivation, itself based on consideration of the space between randomly packed monosized spheres and its evolution as packed powders are densified. Taking then the struts as simplified resistor or bending beam elements, values and trends for $n$ and $N$ emerge that are consistent with experiment. The predictions for conductivity also reproduce Archie's law from soil science.

\section{Derivation}

To understand the geometry of replicated microcellular solids and how it evolves with $V_{\mathrm{m}}$, we consider an assembly of deformable spherical particles (space-holders), all having the same radius. The particles can pack together to a maximum packing density $\phi_{\mathrm{o}} \approx 0.64$ while maintaining their spherical shape. To reach higher packing densities $\left(\phi>\phi_{\mathrm{o}}\right)$ without changing their volume, the densely packed particles must deform. If we evolve the particle assembly to higher density, $\phi>\phi_{\mathrm{o}}$, letting the particle centres approach one another in roughly homothetic fashion, then the particles must deform at their contact points. At the same time, the average number $Z$ of particle-to-particle contacts per particle increases, because new contacts are created as densification brings particle centres closer together. This evolution was elucidated by Arzt and coauthors [36-38].

Between the particles is a volume of interconnected porosity composed of two basic building blocks: (i) narrow channels connected at (ii) wider open pores, which we refer to as 'channels' and 'nodes', respectively. The narrow channels are circumscribed by three contacting particles. Nodes are often defined by the open space between four touching particles, where four channels meet; however, at lower particle density (meaning as $\phi$ nears $\phi_{\mathrm{o}}$ ), some nodes are thicker than the space between four touching spheres. Such thicker nodes comprise the flat regions between two particles that are the nearest neighbours but are not in contact; a few larger nodes of this kind are sketched in 2-D in Fig. 1 and are visible in replicated aluminium structures shown in Fig. 2.

In the replication process this pore network is infiltrated by what will become the solid making the open-pore microcellular material. After the space-holder particles are removed one is left with a microcellular solid composed of the same two elementary building blocks described above, except that these refer to solid material, Fig. 2: (i) channels have become narrow 'struts' connecting at (ii) thicker nodes, ideally, but not always, four struts per node.

If we now assume that the Coxeter identity (strictly valid only for dry foams) [39] can be applied to the network of lines running midway through all channels and nodes delineated by the packed particles, we have:

$Z=\frac{12}{6-\langle n\rangle}$

where $\langle n\rangle$ is the average number of channels bounding one particle contact in the particle assembly. Since a channel is

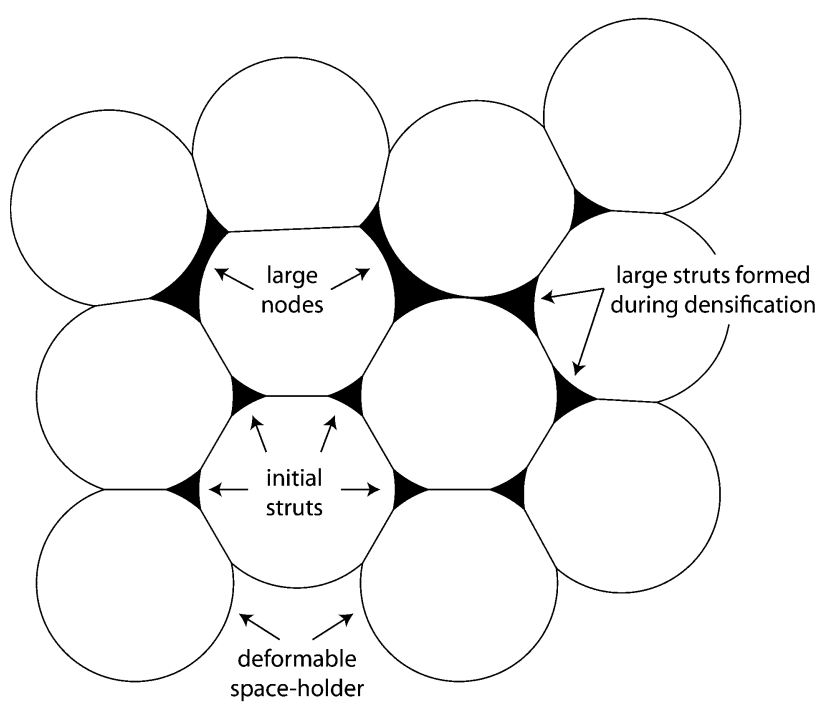

Fig. 12 D schematic of the space between close-packed and partly densified spheres 


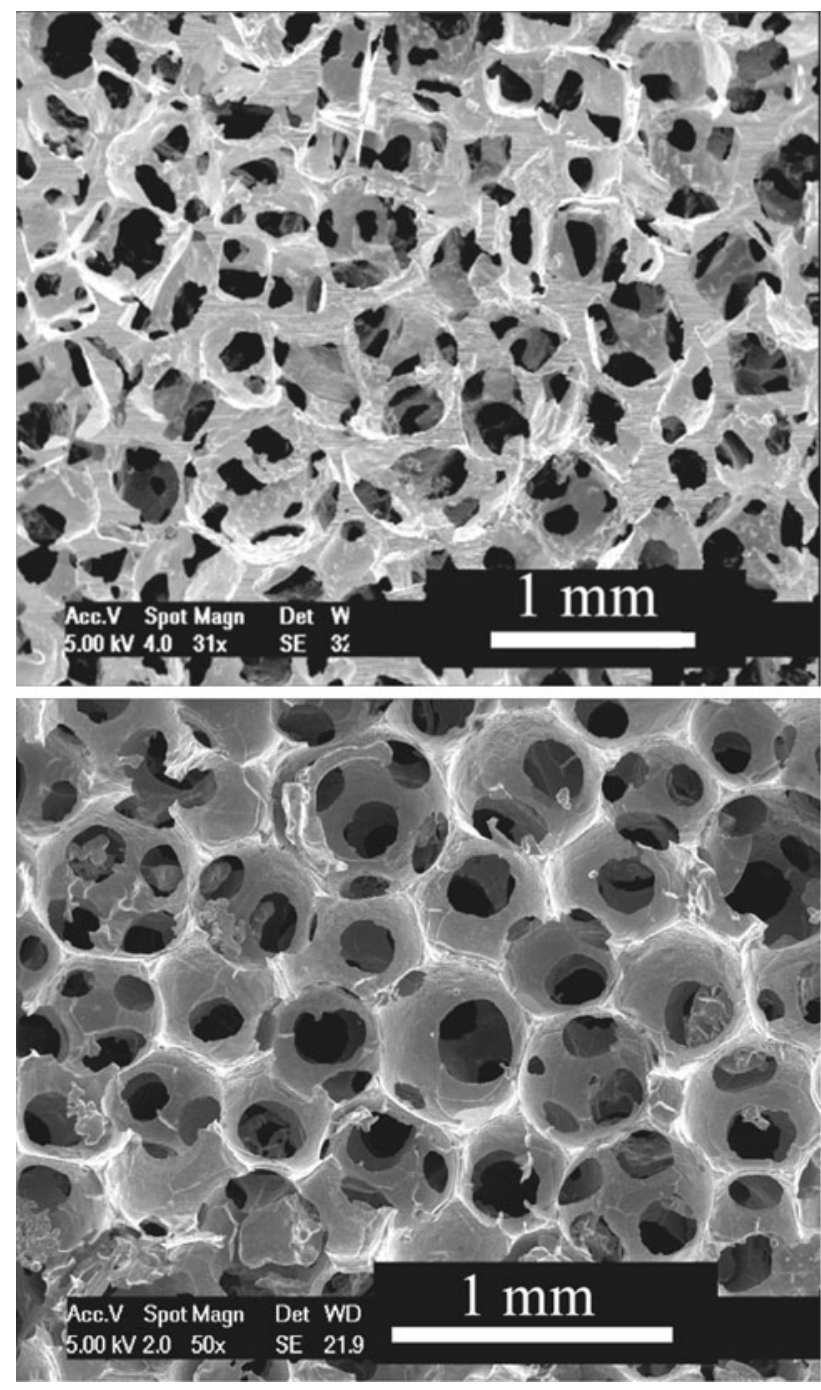

Fig. 2 Replicated foam with $400 \mu \mathrm{m}$ pore of irregular polygonal shape (top) and spherical shape (bottom)

shared by two contacts for each particle, the total number of channels surrounding one particle, $Y$, equals $Z\langle n\rangle / 2$. Hence:

$Y=3(Z-2)$.

When particles first touch, there are roughly $Z=7$ contacts between these; as compaction proceeds, $Z$ increases to reach a maximum value of 14 when $\phi$ approaches unity [36, 40]. $Y$ thus correspondingly evolves from 15 to 36 as the density of particles increases from $\phi_{\mathrm{o}}$ to 1 (Eq. 2); however, in practice the peak value of 36 is not reached since replicated foam structures are seldom produced to have solid fractions below $5 \%$ (in Ref. [41] a few examples are given of replicated microcellular aluminium in this low range of densities). The reason is that as $\phi$ reaches values around $90-95 \%$, pores tend to close-off and spheroidize in powder compacts under the action of surface forces [42-45]. The number of particle contacts $Z$ will thus not exceed 12 in practice, and $Y$ reaches a maximum around 30 .

Thus, at least half of all channels in a particle compact were present initially, from the moment the particles were packed, being delineated between particles that touched in the powder compact at the limit of random dense packing, i.e. before particles deformed to densify the compact. Remaining channels have appeared later, during densification, around newly created particle-to-particle contacts.

Replicated foam mesostructures are, thus, more complex than regular periodic structures assumed in nearly all models proposed so far in the literature. Struts (replicated channels) have unequal cross sections and vary in number, width and length as the foam relative density varies; so do nodes, the number and shape of which also evolve with relative density. Figure 2 gives two examples of replicated aluminium mesostructures; close examination of this figure, and of the more comprehensive views of spherical pore replicated aluminium mesostructures offered in Figs. 7 and 8 of Ref. [35], clearly show this.

Now, (i) the thinnest of all struts are those that surround initial particle-to-particle contacts in the (monosized spherical) powder compact that served to produce the material and (ii) these 'initial' struts always represent at least half of all struts present, as suggested by the calculation above. The threshold for bond percolation in threedimensional networks with the coordination expected for the space between packed spheres (four) is $p_{\mathrm{c}}=0.39$ [46, 47]. This is also the transition threshold from zero to finite values for $C$, and for $E$ in regular networks of coordination four when both stretching and bending of bonds oppose deformation [48-51]. Hence, although thicker struts will influence the conductivity and stiffness of replicated microcellular strut networks, these can reasonably be expected either to form isolated clusters (because they appear around newly formed particle/pore contact points), or to be just past the percolation threshold (at the lowest replicated microcellular material relative densities, near $5 \%$ ). Although the reasoning that precedes cannot constitute proof (if only because, at variance with percolation models, 'initial' and other struts are not distributed here completely at random), it is reasonable to expect that the thinner 'initial' struts exert a dominant influence on both conduction and deformation of the strut-node network composing the open-cell structure of replicated microcellular materials. So we pose this as our starting assumption and seek to deduce the scaling relations that it implies.

'Initial' struts in replicated structures have a cross section defined by the open space left between three initially touching space-holder particles. Their cross-sectional area, $a$, is proportional to the square of some characteristic dimension, say the side of their near-triangular cross 
section $d$, Fig. 3. As the powder compact densifies, the centre-to-centre distance of space-holder particles decreases. If we assume that particle centres remain distributed similarly in space (or in other words that the particle network shrinks homothetically), then if $D$ is the average centre-to-centre separation of initially touching particles, we have:

$\frac{D^{3}}{D_{\mathrm{o}}^{3}}=\frac{\phi_{\mathrm{o}}}{\phi}$

where $D_{\mathrm{o}}$ is the initial particle centre separation distance, equal to twice the initial particle radius.

As the powder compact densifies and $D$ is reduced, the average area defined between each set of three initially contacting particles becomes increasingly narrow. This, in turn, causes channels between the three particles to narrow. If we assume that material within a triangular slice of material defined by the zone of contact of each set of three initially touching particles remains within that slice (and thus leave aside complexities of how the geometry of these channels evolves as they thin), then, as its average crosssectional area is reduced from $(\sqrt{ } 3 / 2) D_{\mathrm{o}}^{2}$ to $(\sqrt{ } 3 / 2) D^{2}$, mass conservation dictates that the average strut crosssectional area (times the thickness of the slice) must be reduced by the same area (times the thickness of the slice). Hence:

$\frac{\sqrt{3}}{4}\left(D_{\mathrm{o}}^{2}-D^{2}\right)=a_{\mathrm{o}}-a=\frac{\sqrt{3}}{4}\left(d_{\mathrm{o}}^{2}-d^{2}\right)$

if we assimilate, for simplicity, the strut cross section and the corresponding slice of surrounding space-holder material to equilateral triangles, Fig. 3. Inserting Eq. 3 this becomes:

$\frac{d^{2}}{D^{2}}=1-\frac{D_{\mathrm{o}}^{2}-d_{\mathrm{o}}^{2}}{D_{\mathrm{o}}^{2}}\left(\frac{1-V_{\mathrm{m}}}{1-V_{\mathrm{m}, \mathrm{o}}}\right)^{2 / 3}$

where $V_{\mathrm{m}}=1-\phi$ is the relative density of the replicated microcellular material produced by infiltration of particles packed to a volume fraction solid of $\phi ; V_{\mathrm{m}, \mathrm{o}} \equiv 1-\phi_{\mathrm{o}}$.

If conduction through the replicated foam is controlled by the rate of flow of current or heat through these initial struts, then the conductivity $C$ of replicated foams will scale as $d^{2} / D^{2}$, leading to:

$C=\alpha\left(1-A\left(1-V_{\mathrm{m}}\right)^{2 / 3}\right)$

with

$A=\frac{D_{\mathrm{o}}^{2}-d_{\mathrm{o}}^{2}}{D_{\mathrm{o}}^{2}}\left(\frac{1}{1-V_{\mathrm{m}, \mathrm{o}}}\right)^{2 / 3}$

Similarly, we take it that elastic deformation of the replicated microcellular material is controlled by bending of these initial struts (the strut coordination being near four, deformation of the structure is expected to be bendingdominated [52]). Then, Young's modulus $E$ (and also the microcellular material shear modulus) will scale as $d^{4} / l^{4}[1]$ where $l$, the length of the struts, is roughly proportional to $D$. $E$ thus scales as $d^{4} / D^{4}$, giving:

$E=\alpha^{\prime}\left(1-2 A\left(1-V_{\mathrm{m}}\right)^{2 / 3}+A^{2}\left(1-V_{\mathrm{m}}\right)^{4 / 3}\right)$

In Eqs. 6 and 8, $\alpha, \alpha^{\prime}$ and $A$ are constants. Their value can be set by:

(i). defining $C_{0}$ and $E_{0}$ as the conductivity and stiffness, respectively, of the replicated microcellular solid at its highest possible relative density, i.e. at $V_{\mathrm{m}}=V_{\mathrm{m}, \mathrm{o}} \equiv 1-\phi_{\mathrm{o}} \approx 0.36\left(C_{0}\right.$ and $E_{0}$ are of course each proportional to the dense solid material values, $C_{\mathrm{m}}$ and $E_{\mathrm{m}}$, respectively) and

(ii). noting that at some point during compaction, channels in the space-holder powder compact pinch-off, causing pores to close and spheroidize (in the theory of powder densification this has been called the transition from Stage 2 to Stage 3 densification [53, 54] or from the intermediate to the final stages of densification [55-57]). Irregularity in the shape and size of 'real' particle preforms causes the moment at which this occurs to vary from strut to strut. In the simplified geometry of the present model, such irregularity is ignored and all initial struts are assumed to pinch-off at a single relative density defined by the ratio of strut to solid matter cross-
Fig. 3 a Cross section of a strut defined between three touching spheres in the initial packed powder compact, and (dotted line) a triangle having the same cross-sectional area; b same after densification
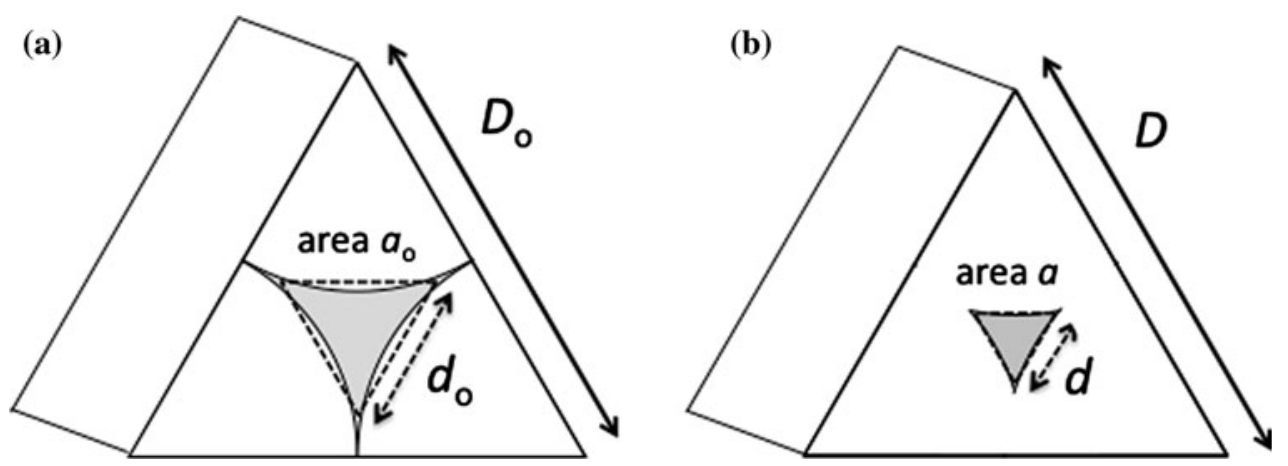
sectional areas in the triangular slice sketched in Fig. 3, and hence by the value of $A$ (Eq. 7).

We thus define $\phi_{\mathrm{c}}=1-V_{\mathrm{m}, \mathrm{c}}$ as the space-holder packing density at which the cross-sectional area of (all) initial struts decreases to zero. Having assumed here that initial struts govern the conduction and elastic stiffness, $V_{\mathrm{m}, \mathrm{c}}$ is the relative density of the microcellular material at which its conductivity or modulus goes to zero; it is also, for the material and processing route at hand, the lowest attainable replicated microcellular material relative density.

Scaling relations (6) and (8) can then be rewritten as:

$$
\begin{aligned}
& \frac{C}{C_{\mathrm{o}}}=\frac{\left(1-\left(\frac{1-V_{\mathrm{m}}}{1-V_{\mathrm{m}, \mathrm{c}}}\right)^{2 / 3}\right)}{\left(1-\left(\frac{1-V_{\mathrm{m}, \mathrm{o}}}{1-V_{\mathrm{m}, \mathrm{c}}}\right)^{2 / 3}\right)} \text { and } \\
& \frac{E}{E_{\mathrm{o}}}=\frac{\left(1-2\left(\frac{1-V_{\mathrm{m}}}{1-V_{\mathrm{m}, \mathrm{c}}}\right)^{2 / 3}+\left(\frac{1-V_{\mathrm{m}}}{1-V_{\mathrm{m}, \mathrm{c}}}\right)^{4 / 3}\right)}{\left(1-2\left(\frac{1-V_{\mathrm{m}, \mathrm{o}}}{1-V_{\mathrm{m}, \mathrm{c}}}\right)^{2 / 3}+\left(\frac{1-V_{\mathrm{m}, \mathrm{o}}}{1-V_{\mathrm{m}, \mathrm{c}}}\right)^{4 / 3}\right)}
\end{aligned}
$$

\section{Results and discussion}

Figure $4 \mathrm{a}, \mathrm{b}$ gives plots, in the usual logarithmic coordinates, of $C / C_{0}$ and $E / E_{0}$ versus $V_{\mathrm{m}}$ for $V_{\mathrm{m}, \mathrm{c}}=0,2.5,5,7.5$ and $10 \%$, taking $V_{\mathrm{m}, \mathrm{o}}=1-\phi_{\mathrm{o}}=0.36$. With $V_{\mathrm{m}, \mathrm{c}}=0$ the two plots return approximately the two straight lines, of slope 1 or 2, respectively, characteristic of fixed-architecture microcellular materials models [1-3]. This does not come out simply from Eqs. 5, 6, 7 and 8 but must be because the assumed geometry then gives, approximately, to initial struts a cross section and a length that both are in keeping with the average material architecture as it evolves with changing $V_{\mathrm{m}}$.

As $V_{\mathrm{m}, \mathrm{c}}$ increases, the curves deviate from the usual scaling relation. These now show a cutoff where both fall precipitously (as should be since the microcellular material loses coherency). At higher relative densities the curves nearly trace a straight line, the slope of which increases as $V_{\mathrm{m}, \mathrm{c}}$ increases.

The simple model presented here thus reproduces the gradual increase in slope that is found as $V_{\mathrm{m}}$ decreases in corresponding experimental plots of $E$ versus $V_{\mathrm{m}}[23,35]$. Furthermore, with $V_{\mathrm{m}, \mathrm{c}}=5 \%$, the slope of the curve is that displayed by experimental data, both for $C$ and for $E$, in replicated aluminium over the range of relative densities traced by experimental data [10, 23, 35]. This value for $V_{\mathrm{m}, \mathrm{c}}$ is reasonable: (i) $5 \%$ is the porosity at which pores are on average documented to close-off in pressed metal powder compacts [44, 45] and (ii) this is the observed lower limit of foam relative densities that can be achieved in replication processing using equiaxed space-holder particles [41].

The calculation presented here is highly simplified: it ignores many effects (for example the fact that initial strut cross sections have varying shapes and areas, or loadsharing between initial struts and thicker struts). Yet, despite its simplicity, it captures the observed scaling behaviour when its one adjustable variable takes its most reasonable value. The main difference between what is presented here and corresponding models proposed so far in the literature is that we take the architecture of the microcellular material to vary with the relative density: struts considered to govern conduction and elastic deformation of the microcellular material do not thin or shorten at the same rate as does, on average, the microcellular material. Rather, we decouple the evolution of elements we view as critical to the property in question from that of the cell architecture as a whole, and evaluate this evolution with no concern for the (highly complex) evolution of the material architecture in other locations, basing the present derivation on the physics of powder compact densification.

The derivation also gives a view to the physical origin of Archie's law: $n \approx 1.5$ is indeed often observed in wet rock (see Table 5.2 of Ref. [15]). Many sedimentary rocks are formed by deposition and compaction ('diagenetic') processes that resemble geometrically preform densification processes [14-17, 20]. The conducting phase in wet rock (ion-containing water), therefore, has a geometry similar to that of the solid in replicated microcellular solids, leading to infer that its conductivity should follow the same law. In fact, although it is much simpler (or, viewed differently, more simplistic), the present derivation is close to the Bernal sphere distribution model offered in Ref. [20] to explain Archie's law as observed in consolidated soil.

We close by pointing out similarities and differences that exist between the problem treated here, and that of the conductivity of liquid foams, or similarly that of emulsions in which only the continuous phase conducts [39, 58, 59]. In all such structures conduction is carried by a phase that fills the space between close random packed deformed spheres. In structures addressed here (replicated microcellular materials; wet soil,...) the spheres are solid and their shape is determined by plastic deformation or by diffusive transport mechanisms that cause the packed solid particles to densify. In wet foams and emulsions, on the other hand, the spheres are soft: they offer essentially no resistance to deformation. Their shape is then entirely dictated by equilibration of capillary and disjoining forces. These tend to equalize strut cross sections across the entire structure, causing in turn the conductivity to remain finite as the volume fraction of conducting phase tends to zero. This is confirmed by experiment: in wet foams and emulsions $C$ follows, at vanishing conducting phase, the Lemlich rule 
Fig. 4 Predicted scaling relations for a the conductivity $C$ and $\mathbf{b}$ Young's modulus $E$ of replicated microcellular structures, as a function of the relative density $V_{\mathrm{m}, \mathrm{c}}$ at which thinner, 'initial' struts close-off (corresponding to the packing density $\phi_{\mathrm{c}}=1-V_{\mathrm{m}, \mathrm{c}}$ at which cylindrical pores in the powder preform close-off and become isolated)

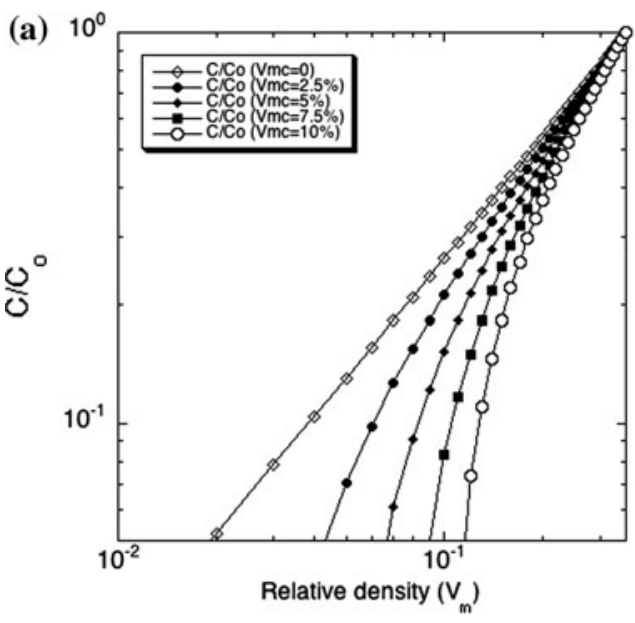

( $k=1 / 3, n=1$ in Eq. 1) [39, 58-60]. The present derivation, therefore, does not apply to liquid foams or emulsions.

\section{Conclusion}

In summary, a simple calculation shows that scaling exponents, around 1.5 and 3, respectively, displayed by the conductivity and Young's modulus of replicated microcellular materials are explained if one assumes that these properties are controlled by struts, the shape of which evolves with $V_{\mathrm{m}}$ differently from that of the material as a whole. The derivation also offers a simple alternative explanation of what is known in soil science as Archie's law.

Acknowledgement This work was funded by the Swiss National Science Foundation, Project No. 200020-1182134. The authors thank Dr. Russell Goodall (formerly of our laboratory and now at the University of Sheffield) for the SEM micrograph of replicated microcellular aluminium with spherical pores, and Drs. Russell Goodall, Luc Salvo and Ariane Marmottant for many stimulating discussions on the topic of this contribution.

\section{References}

1. Gibson LJ, Ashby MF (1997) Cellular Solids: Structure and Properties, 3rd edn. Cambridge University Press, Cambridge

2. Ashby MF, Evans A, Fleck NA, Gibson LJ, Hutchinson JW, Wadley HNG (2000) Metal foams: a design guide. Butterworth Heinemann, Boston

3. Goodall R, Mortensen A (2013) In: Hono K, Laughlin D (eds) Physical metallurgy, 5th edn. Elsevier, Amsterdam

4. LeMay JD, Hopper RW, Hrubesh LW, Pekala RW (1990) MRS Bull 15:19

5. Fitzgerald TJ, Michaud VJ, Mortensen A (1995) J Mater Sci 30:1037. doi:10.1007_BF01178442

6. San Marchi C, Mortensen A (2002) In: Degischer HP, Kriszt B (eds) Handbook of Cellular Metals. Wiley-VCH Verlag, Weinheim
7. Conde Y, Despois JF, Goodall R et al (2006) Adv Eng Mater 8:795

8. Combaz E, Bacciarini C, Charvet R, Dufour W, Dauphin F, Mortensen A (2010) Acta Mater 58:5168. doi:10.1016/j.actamat. 2010.05.053

9. Prieto R, Louis E, Molina JM (2012) Carbon 50:1904. doi:10. 1016/j.carbon.2011.12.041

10. Goodall R, Weber L, Mortensen A (2006) J Appl Phys 100: 044912

11. Kovacik J (1998) Scripta Mater 39:153

12. Weber L, Dorn J, Mortensen A (2003) Acta Mater 51:3199

13. Weber L, Fischer C, Mortensen A (2003) Acta Mater 51:495

14. Sen PN, Scala C, Cohen MH (1981) Geophysics 46:781

15. Hunt A, Ewing R (2009) Percolation theory for flow in porous media. Springer, Heidelberg

16. Sahimi M (1993) Rev Mod Phys 65:1393

17. Sahimi M (2011) Flow and transport in porous media and fractured rock: from classical methods to modern approaches. Second, Revised and Enlarged Edition. Wiley-VCH Verlag, Weinheim

18. Yonezawa F, Cohen MH (1983) J Appl Phys 54:2895

19. Wong P-Z, Koplik J, Tomanic JP (1984) Phys Rev B 30:6606

20. Roberts JN, Schwartz LM (1985) Phys Rev B 31:5990

21. Kovacik J, Simancik F (1998) Scripta Mater 39:239

22. Torquato S (2002) Random heterogeneous materials: microstructure and macroscopic properties. Springer-Verlag, New York

23. Despois JF, Mueller R, Mortensen A (2006) Acta Mater 54:4129

24. Warren WE, Kraynik AM (1988) J Appl Mech 55:341

25. Warren WE, Kraynik AM (1997) J Appl Mech 64:787

26. Zhu HX, Knott JF, Mills NJ (1997) J Mech Phys Solids 45:319

27. Zhu HX, Hobdell JR, Windle AH (2000) Acta Mater 48:4893. doi:10.1016/s1359-6454(00)00282-2

28. Gong L, Kyriakides S, Jang WY (2005) Int J Solids Struct 42:1355

29. Jang WY, Kraynik AM, Kyriakides S (2008) Int J Solids Struct 45: 1845

30. Li K, Gao XL, Roy AK (2003) Compos Sci Technol 63:1769. doi:10.1016/s0266-3538(03)00117-9

31. Sihn S, Roy AK (2004) J Mech Phys Solids 52:167. doi:10.1016/ s0022-5096(03)00072-3

32. Rossoll A, Mortensen A (2013) Scripta Materialia 68:44. doi:10. 1016/j.scriptamat.2012.05.048

33. Roberts AP, Garboczi EJ (2001) Acta Mater 49:189

34. Roberts AP, Garboczi EJ (2002) J Mech Phys Solids 50:33

35. Goodall R, Marmottant A, Salvo L, Mortensen A (2007) Mater Sci Eng A 465:124. doi:10.1016/j.msea.2007.02.002 
36. Arzt E (1982) Acta Metall 30:1883

37. Fischmeister HF, Arzt E (1983) Powder Metall 26:82

38. Arzt E, Ashby MF, Easterling KE (1983) Metall Trans 14A:211

39. Weaire D, Hutzler S (1999) The physics of foam. Oxford University Press, Oxford

40. Weaire D (2002) Adv Eng Mater 4:723

41. Amsterdam E, Goodall R, Mortensen A, Onck PR, De Hosson JTM (2008) Mater Sci Eng A 496:376. doi:10.1016/j.msea.2008. 05.036

42. Larson RG, Scriven LE, Davis HT (1981) Chem Eng Sci 36:57

43. Helle AS, Easterling KE, Ashby MF (1985) Acta Metall 33:2163

44. Ashby MF (1987) In: Garware T (ed) HIP: proceedings of the international conference on hot isostatic pressing. Centek Publishers, Luleå

45. Jones WD (1960) Fundamental principles of powder metallurgy. Edward Arnold, London

46. Stauffer D, Aharony A (1994) Introduction to percolation theory, revised second edition. CRC Press, Boca Raton

47. Sahimi M (1994) Applications of percolation theory. Taylor and Francis, London
48. Halperin BI, Feng S, Sen PN (1985) Phys Rev Lett 54:2391

49. Feng S, Halperin BI, Sen PN (1987) Phys Rev B 35:197

50. Sahimi M, Arbabi S (1993) Phys Rev B 47:703

51. Sahimi M (1996) Chem Eng J Biochem Eng J 64:21. doi:10.1016/ S0923-0467(96)03103-X

52. Deshpande VS, Ashby MF, Fleck NA (2001) Acta Mater 49:1035

53. Ashby MF (1974) Acta Metall 22:275

54. Beere W (1975) Acta Metall 23:139

55. German RM (1996) Sintering theory and practice. Wiley, New York

56. Kang SJL (2005) Sintering: densification, grain growth and microstructure. Elsevier, Butterworth Heinemann, Amsterdam

57. Coble RL (1961) J Appl Phys 32:787

58. Phelan R, Weaire D, Peters EAJF, Verbist G (1996) J Phys 8:L475

59. Feitosa K, Marze S, Saint-Jalmes A, Durian DJ (2005) J Phys 17:6301

60. Lemlich R (1978) J Colloid Interface Sci 64:107 